\title{
A critical overview of four-stage model under the background of the rise of ride-sharing
}

\author{
MENG Xintong ${ }^{1}$ \\ ${ }^{1}$ School of Economics and Management Beijing Jiaotong University Beijing, China
}

\begin{abstract}
Future transport systems are expected to rely to a much greater extent on intelligent ride-sharing services, thus ride-sharing will have a significant impact on traffic planning and demand forecasting. This paper aims to review the classic transport planning four-stage model critically and extend it to make it more appropriate for traffic planning in the context of the rise of ride-sharing.
\end{abstract}

\section{Introduction}

\subsection{Four-stage transport planning model}

In practice in the $1960 \mathrm{~s}$, a classic transport model, the four-stage transport planning model, has gradually formed, the general form of which is shown in Figure 1. This model is based on zones and networks of the research area, and performs based on a series of collected planning data, calibration data and validation data.

The first step of the four-stage model is called trip generation, the basic two tools for which are multiple linear regression and category analysis (cross classification). The aim of trip generation is to estimate the number of trips generated and attracted by each zone over a given period of time. The main goal of the second stage trip distribution is the determination of the O-D pairs, allocating these trips to specific destinations and generating an O-D trip matrix. The two popular methods of this stage are growth factor methods and gravity models, which can be combined with each other. Next, the purpose of the third stage modal split is to estimate the proportions of trips by the different modes of transport, using the probit method and logit method as the two common methods. That is, the trips in the matrix are assigned to different modes. Finally, the purpose of the last stage assignment is to assign trips of various travel modes to the corresponding networks.

\subsection{Ride-sharing}

"Ride-sharing" have a variety of interpretations, but "intelligent ride-sharing" is defined as a system based on web or application, which can automatically "match" travel requests from more than one travellers so that they can complete the trip in a car, either for each traveller's complete journey, or to/from another mode. One of them may or may not be the driver. Richter divided ride-sharing into two categories ${ }^{[9]}$. One is called direct ride-sharing, referring to ride-sharing is the only mode of the whole trip, and the other is called intermodal ride-sharing, "where ride-sharing acts as a feeder system for the first/last mile of a combined trip with public transport" ${ }^{[9]}$. Ride-sharing has the attributes of both public and private transport especially in the intermodal ride-sharing form.

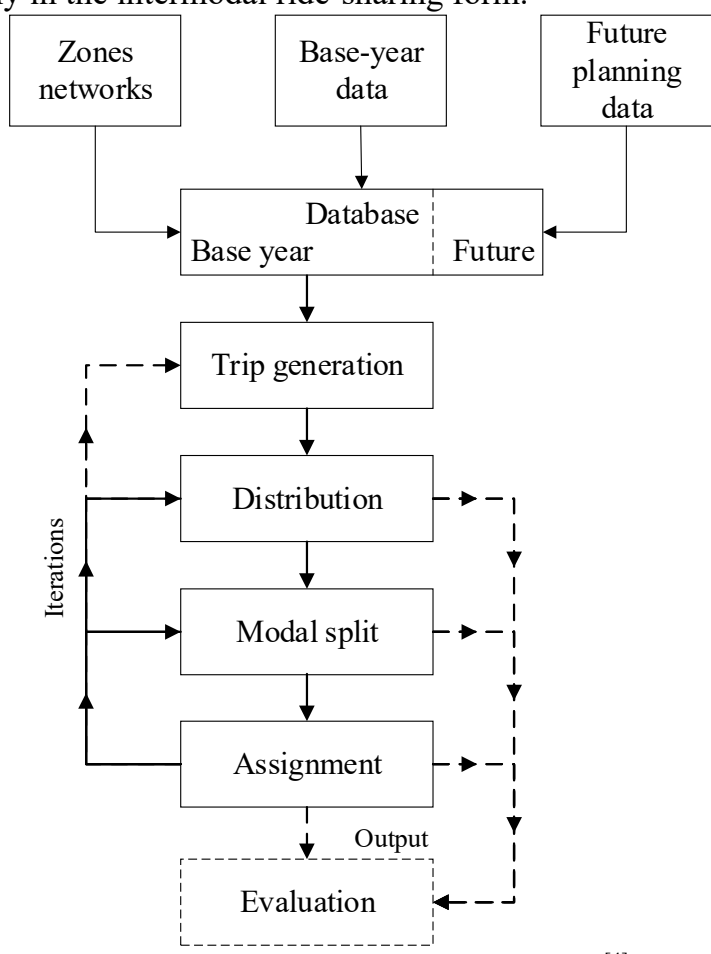

Figure 1 The structure of four-stage transport model ${ }^{[4]}$

Ride-sharing aims to reduce vehicle kilometers by centralizing trips for different people in one car. The growth of ride-sharing could create significant social and environmental benefits by reducing the number of cars used for personal travel and increasing the use of available seats. Ride-sharing also affects the demand for travel by changing the supply of transport. As the literature linking ride-sharing to the four-stage model is scarce so far, it is necessary to integrate ride-sharing services into four-stage models. To quote, "as ride-sourcing becomes more 
popular, it may have a greater impact on travel and therefore demand models should consider how to include this in the four step process" [14]. Therefore, in order to evaluate the impact of traffic supply changes accurately, it is necessary to regard ride-sharing as an emerging alternative mode and expand the four-stage transport model under this circumstance.

The aim of this paper is to make a critical review of the typical four-stage model under the background of the rise of ride-sharing to see which features of ride-sharing can be captured by or missing from the four-stage model, thereby modifying and supplementing the four-stage model. In general, the features that can affect the earlier stage may also affect one or more stages after that stage. For example, Zhen mentioned that except the direct impact of one factor on assignment step, "changes came with ridesourcing in trip generation and mode split also impact traffic assignment" ${ }^{[14]}$

\section{Features of ride-sharing that could be captured with the four-stage model}

\subsection{Alleviation of traffic congestion and travel efficiency improvement}

Some previous studies show that ride-sharing contributes a lot to alleviating congestion and emissions reduction. Yin et al. conducted a case study in Paris region to assess the effect of ride-sharing on reducing traffic congestion and emission by sensitivity analysis ${ }^{[12]}$. They concluded that ride-sharing significantly reduced the use of private cars, thus reducing traffic congestion - "The maximum reduction is around $40 \%$ when ride-sharing growth being $100 \%$ for all the Paris region". Lokhandwala and Cai compared traditional taxis to shared autonomous taxis and found that when holding service level unchanged and shifting trip mode from the former to the latter, the fleet size would reduce by $59 \%{ }^{[7]}$. Additionally, other benefits of ride-sharing in Lokhandwala and Cai also discovered its contribution to improving traffic efficiency: the improvement in occupancy (from 1.2 to 3) meant increased vehicle use efficiency, and the reduction in total travel distance and carbon emissions meant a significant contribution to energy conservation and emission reduction ${ }^{[7]}$

Reduction of car use and alleviation of traffic congestion means improved travel efficiency. On the one hand, people may be willing to take those trips which are originally dispensable, which is first reflected in the generation stage and may affect every subsequent stage of classic four-stage model. On the other hand, the improved travel efficiency may lead to a shorter travel time for the same trip compared to the original, but it is currently unable to be directly captured by the four-stage planning model and this issue will be discussed in the next chapter.

\subsection{Change in mode share}

The most immediate impact of the emergence of ridesharing is on mode share of transport, which has been illustrated in a number of studies.

Moreno et al. argued that the share of intermediate transport modes, like ride-sharing, was increasing at the expense of a reduction of mode share of cars, public transport and taxis ${ }^{[8]}$. In the case of Yin et al. for the Paris region, it was found that part of the trips by public transport and soft mode (i.e. walking and cycling) were attracted by private cars with the development of ridesharing ${ }^{[12]}$. The survey of Zhen illustrated the results that "ride-sourcing trips were shifted from the different traditional types of modes considered in the mode choice models" [14]. Chung et al. indicated that ride-sharing had a significant reducing effect on on-road traffic, so it was believed that ride-sharing would not compete with, but complement public transport ${ }^{[3]}$. In the analysis China's ride-sharing services, Dong et al. believed that ridesharing was a complement transport mode of taxi, which could meet part of the traffic demand ${ }^{[5]}$. Zhang and Zhang illustrated in the findings that the frequency of ridesharing increased by $7.9 \%$ and the probability of traveling by ride-sharing increased by $23 \%$ significantly for onevehicle reduction in households, which confirmed the relationship between ride-sharing and transport mode change from the perspective of vehicle ownership ${ }^{[13]}$.

The impact of ride-sharing on modal split stage can be captured by the four-stage model, that is, the reduction of the existing traditional modes share (i.e. soft modes, public transport and typical private cars) can be reflected. But the impact of ride-sharing on the third stage goes far beyond that. Some studies believe that ride-sharing can be included in the list of modes as a new alternative, especially as an important feeder system for the "first/last mile" in the future. Zhen concluded that in travel demand models, ride-sourcing, including ride-sharing, could be considered as a new mode of transport or categorised as an automatic or transit mode with new characteristics ${ }^{[14]}$. Thus travellers get new alternative mode choice. Richter also indicated that the existing steps of the four-stage model were supposed to be modified when integrating ride-sharing services into four-stage travel demand models [9]. The modification of the model depends on the form of ride-sharing, i.e. direct ride-sharing and intermodal ridesharing. As the former, it is also suggested adding ridesharing to the mode choice set as a new travel mode, while the latter offers a new option in public transport supply.

Although plenty of evidence for ride-sharing's effect on mode exists, this effect is still limited because some people have restrictions on their ride-share participants. As Xiong mentioned, the more restrictions a ride-share user imposed on the ride-share participant (such as gender, habit, familiarity), the less likely the user was to succeed in matching with others ${ }^{[11]}$.

\subsection{Change in route choice}

As a new alternative mode of public transport feeder system, ride-sharing also has a significant influence on route choice. Richter indicated that the route choice alternatives in the public transport supply would be altered by ride-sharing [9]. The private car travellers who have their own travel habits and regular routes may change a lot 
in route choice due to ride-sharing. As a result, public may have greatly different requirements on the capacity of existing routes than before, and/or new routes may need to be built.

Many studies aimed to design algorithms and establish more efficient models technically so that automatic matching and vehicle route choice could be optimised ${ }^{[2,10]}$. This also indicates that the emergence of ride-sharing promotes the optimisation of route choice, thus affecting the transport planning, which will be captured by the fourth stage assignment.

\subsection{Change in travel frequency}

A number of studies are on the relationship between ridesharing and travel frequency. On the one hand, studies have shown that the emergence of ride-sharing will increase the travel rate of people. For example, Zhen illustrated that when putting ride-sourcing into consideration, it led to a result that the trip rate per household, which represented the average number of trips per household for a feature category, was higher ${ }^{[14]}$. On the other hand, a successful ride-sharing requires a number of ride-share participants (i.e., a high population density) to achieve a high enough ride-sharing frequency of use. Thus ride-sharing may make an originally attractive area more attractive than others. "Attractive" here refers to more complete infrastructure, more social functions, and great ability to attract people, such as the prosperous downtown areas. The reason is that more attractive regions are more likely to succeed in matching ride-share participants, so people will choose to request their ridesharing trips in these regions. This behavioral response is reflected in the trip generation and subsequent stages. For example, the number of trips of some O-D pairs may increase (or decrease), which will affect the mode share and traffic assignment on corresponding routes.

Zhang and Zhang examined how household vehicle ownership was related to the frequency and probability of using ride-sharing as a transport mode and determined how this relationship changes with population density ${ }^{[13]}$. One of the results was that people living in densely populated areas and cities tend to use ride-sharing services more frequently than those living in a region with lower population density and urban areas, which supported the view above.

\subsection{Travel cost-sharing}

As mentioned in Agatz et al., one of the features of ridesharing is cost-sharing ${ }^{[2]}$. The emergence of ride-sharing allows the variable (and even fixed) costs associated with travel to be distributed among ride-share participants, which is beneficial to all participants from the perspective of travel cost savings. In the case study of Yin et al., the travel cost in Paris region was reduced by sharing the travel costs of the ride-sharing vehicles ${ }^{[12]}$. The lower travel cost may make private cars more attractive to people, thus affecting the share of each mode.

Some studies focus on determining appropriate pricing schemes to optimise travel cost of ride-sharing so that it has a price advantage over other travel modes, especially solo private car driver, thus attracting more private car drivers to become ride-share participants ${ }^{[6]}$. The change in modes can be captured by modal split stage and the subsequent assignment stage of classic four-stage model.

\section{Features of ride-sharing that are missing from four-stage model}

The four-stage model is regarded as focusing on a limited range of traveller responses, so analysis of broader responses to transport problems and solutions is needed. Facing the emergence of ride-sharing, travellers can respond with a series of simple behavioral changes. However, these behavioral responses may not be captured by the four-stage model, so the appropriate extension of this transport planning model is necessary in the context of ride-sharing's development.

An important element missing from the four-stage planning model is the impact of time on transport planning. One of the features of ride-sharing is non-recurring trips and more flexible, which determines that it is different from carpooling ${ }^{[2]}$. On the one hand, it is not limited by rigid schedule and travel frequency. On the other hand, it also enhances the uncertainty of matching and travel time. The following is a discussion about the extension of fourstage model from the perspectives of departure time and travel time.

\subsection{Uncertainty of departure time}

Agatz et al. pointed out that ride-sharing had a feature: dynamic ${ }^{[2]}$. People can create ride-sharing travel requests at any time through the App on smartphones and be matched automatically after a while. Since the waiting time can range from a few minutes to a few hours, it may affect people's departure time, which is missing from the four-stage model.

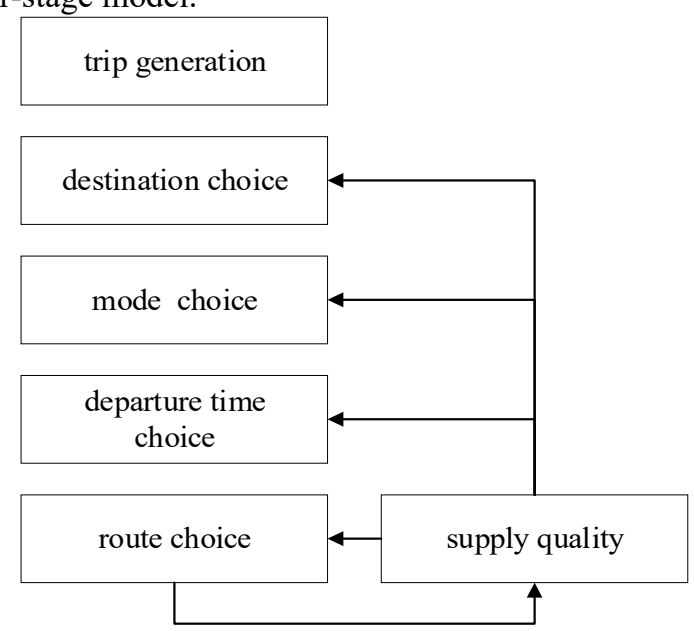

Figure 2 Four-stage algorithm supplemented by departure time choice ${ }^{[9]}$

Some literature has taken departure time into account as an important element when designing models or schemes. Agatz et al. asked participants to provide the earliest possible departure time and the latest arrival time ${ }^{[1]}$. Liu and Li explicitly pointed out that traffic congestion 
would change over time and they designed the commuters' ride-sharing pricing scheme based on this ${ }^{[6]}$. Yin et al. found that the reducing effect of ride-sharing on UPV and traffic congestion was different during morning peak and evening peak, which might also affect people's choice of departure time ${ }^{[12]}$. Therefore, the departure time choice seems to be important in transport planning, which determines the road conditions and the congestion level faced by travellers, thus affecting the traffic assignment. This paper refers to the extension of the four-stage model in Richter and adds a "departure time" stage between modal split and assignment stages, turning it into a "fivestage model" (see Figure 2) ${ }^{[9]}$.

\subsection{Uncertainty of travel time}

Some literature confirmed the influence of ride-sharing on travel time. Richter indicated that the travel times in the public transport supply would be altered by the emergence of ride-sharing ${ }^{[9]}$. Yin et al. conducted a case study in Paris region and discovered that ride-sharing reduces travel time by increasing the average travel speed in the traffic assignment, which was beneficial for the ride-sharing choice ${ }^{[12]}$. Thus it is necessary to take travel time into account in traffic planning, as this may affect traffic assignment in different time periods.

\section{Conclusion}

In general, ride-sharing affects the transport system in many ways since it becoming considerable as a new transport mode. This paper focuses on the features of ridesharing and its impact on transport planning from the aspects of travel efficiency, mode share, route choice, travel frequency, travel cost, departure time and travel time. Plus, this paper provides a critical review of the fourstage model in the context of the rise of ride-sharing. On this basis, this paper attempts to extend and integrate ridesharing into the classic four-stage model.

Definitely, people's behavioral responses to the rise of ride-sharing are not limited to those mentioned above. To quote, "other more complex responses take place in the longer term, for example changes in jobs, residential location and choice of shopping areas and so on; all of these will respond, at least partially, to changes in the accessibility provided by the transport system" ${ }^{[4]}$. If the features and impacts of these behavioral responses cannot be captured by the classic four-stage model, it still needs a further extension to make more accurate demand forecasts for transport planning.

\section{References}

1. Agatz, N., Erera, A. L., Savelsbergh, M. W., and Wang, X. 2011. Dynamic ride-sharing: A simulation study in metro Atlanta. Procedia-Social and Behavioral Sciences. 17, pp.532-550.

2. Agatz, N., Erera, A., Savelsbergh, M. and Wang, X. 2012. Optimisation for dynamic ride-sharing: A review. European Journal of Operational
Research. 223(2), pp.295-303.

3. Chung, C. L., Jeng, J. Y., Lee, Z. Y., and Lee, J. S. 2012. Study of carpool user behaviors and route characteristics in Taiwan. In Sustainable Transportation Systems: Plan, Design, Build, Manage, and Maintain. pp. 356-363.

4. de Dios Ortuzar, J. and Willumsen, L.G. 2011. Modelling transport. $5^{\text {th }}$ ed. John wiley \& sons.

5. Dong, Y., Wang, S., Li, L., and Zhang, Z. 2018. An empirical study on travel patterns of internet based ride-sharing. Transportation research part $C$ : emerging technologies. 86, pp.1-22.

6. Liu, Y., and Li, Y. 2017. Pricing scheme design of ride-sharing program in morning commute problem. Transportation Research Part C: Emerging Technologies. 79, pp.156-177.

7. Lokhandwala, M., and Cai, H. 2018. Dynamic ride sharing using traditional taxis and shared autonomous taxis: A case study of NYC. Transportation Research Part C: Emerging Technologies. 97, 45-60.

8. Moreno, A. T., Michalski, A., Llorca, C., and Moeckel, R. 2018. Shared autonomous vehicles effect on vehicle-km traveled and average trip duration. Journal of Advanced Transportation. 2018.

9. Richter, E., Friedrich, M., Migl, A. and Hartleb, J. 2019. Integrating ride-sharing services with automated vehicles into macroscopic travel demand models. In 2019 6th International Conference on Models and Technologies for Intelligent Transportation Systems (MT-ITS). pp.1-8.

10. Santos, D. O., and Xavier, E. C. 2015. Taxi and ride sharing: A dynamic dial-a-ride problem with money as an incentive. Expert Systems with Applications. 42(19), pp.6728-6737.

11. Xiong, C., Hetrakul, P., and Zhang, L. 2014. On ridesharing: a departure time choice analysis with latent carpooling preference. Journal of Transportation Engineering. 140(8): 04014033.

12. Yin, B., Liu, L., Coulombel, N., and Viguié, V. 2017. Evaluation of ride-sharing impacts using an integrated transport land-use model: a case study for the Paris region. Transportation Research Procedia. 27, pp.824-831.

13. Zhang, Y., and Zhang, Y. 2018. Examining the relationship between household vehicle ownership and ride-sharing behaviors in the United States. Sustainability. 10(8): 2720.

14. Zhen, C. 2015. Impact of ride-sourcing services on travel habits and transportation planning. Doctor. dissertation, University of Pittsburgh. 